\title{
Existential Quantification for Variant Ownership
}

\author{
Nicholas Cameron^ and Sophia Drossopoulou \\ Imperial College London \\ \{ncameron, scd\}@doc.ic.ac.uk
}

\begin{abstract}
Ownership types characterize the topology of objects in the heap, through a characterization of the context to which an object belongs. They have been used to support reasoning, memory management, concurrency, etc. Subtyping is traditionally invariant w.r.t. contexts, which has often proven inflexible in some situations. Recent work has introduced restricted forms of subtype variance and unknown context, but in a rather ad-hoc and restricted way.

We develop $J_{0} \exists$, a calculus which supports parameterisation of types, as well as contexts, and allows variant subtyping of contexts based on existential quantification. Jo $\exists$ is more expressive, general, and uniform than previous works which add variance to ownership languages. Our explicit use of existential types makes the connection to type-theoretic foundations from existential types more transparent. We prove type soundness for $\mathrm{Jo} \exists$ and extend it to $\mathrm{Jo}_{\mathrm{o}} \exists_{\text {deep }}$ which enforces the owners-as-dominators property.
\end{abstract}

\section{Introduction}

Ownership types 91011 support a characterization of the topology of objects in the heap. They have been successfully applied in many areas. Boyapati 3 et al. annotated several Java library classes and multithreaded server programs, effectively preventing data races. Vitek et al. used ownership types to support memory management in real time systems, with applications such as flying unmanned aircraft [2], while Aldrich et al. used ownership to enforce software architectures in large, real-world software [1].

Usually ownership types are expressed by classes parameterised by formal context parameters, e.g., class $\mathrm{C}<01,02, \circ 3>\{\ldots\}$, and types parameterised by actuals, e.g., $\mathrm{C}<$ this, $02,02>$. Context parameters represent objects. The first context parameter denotes the owner of the corresponding object. We say that an object is inside its owner, and all transitive owners of the latter. This implicitly defines a tree structure of owners in the heap.

Deep ownership systems enforce the owners-as-dominators property [119], which requires that the path to any object o from the root object passes through the owner of o. That is, objects are dominated by their owners. Such encapsulated objects are protected from direct and indirect access.

\footnotetext{
* Author's current address is Victoria University of Wellington, New Zealand. 
In many variations of ownership types [9 10222 23], actual context parameters must be known and invariant: they must not vary with execution or subtyping; i.e., $\mathrm{C}<01>$ is not a subtype of $\mathrm{C}<02>$ even if 01 is inside 02 . This follows generic types: List<Dog> is not a subtype of List<Animal>.

Recent work on ownership types has introduced the concept of unknown, flexible contexts: universe types [13] support the annotation any, MOJO [6] uses the context parameter ?, and effective ownership 19 uses an any context. These unknown owners introduce variant subtyping, whereby, e.g., $\mathrm{C}<\mathrm{o}>$ is a subtype of $\mathbf{C}<$ ? $>$. Variant ownership types [18] support variance annotations to more precisely describe variance properties of ownership types.

All these systems are somewhat ad hoc in formalisation - there is no direct link to the underlying theory of existential types. In particular, they do not support:

1 two or more context parameters are unknown, but known to be the same, e.g., in the type $\exists$ ○. C<०, o>;

2 context polymorphic methods 923 in the presence of variant contexts;

3 upper and lower bounds on variant contexts;

4 scoping of unknown contexts, e.g., to distinguish a list of students which may have different owners, from a list of student which share the same unknown owners, i.e., List<this, $\exists$ o.Student<૦>>, and $\exists$ o. List<this, Student<૦>>.

To bridge this gap, we develop $\mathrm{J}_{\mathrm{o}} \exists$, which has its foundations in existential types and supports all these features. $\mathrm{Jo}_{\mathrm{O}} \exists$ is a purely descriptive system, in that it only describes the heap topology, and guarantees that the topology is preserved, but does not restrict the topology in any way. We then develop a flavour of $\mathrm{J}_{\mathrm{O}} \exists$, called $\mathrm{J}_{\mathrm{o}} \exists_{\text {deep }}$, which also supports deep ownership. We have distinguished deep ownership from the existential aspects, because descriptive ownership systems are useful in their own right (e.g., to support reasoning with effects).

$\mathrm{Jo} \exists$ is a foundational, rather than usable, system. We expect it to be useful to reason about variance in ownership systems and to compare the various implementations of ownership variance. Whilst it is expressive and powerful, Jo $\exists$ is verbose. Practical adoption of $\mathrm{J}_{\mathrm{O}} \exists$ would require heavy syntactic sugaring.

Recent work with Java wildcards and similar systems 751620 has used existential types to implement and formalise subtype variance in object-oriented languages. In these systems existential types are often implicit 2016], a more programmer-friendly syntax obscures the underlying existential types. Packing and unpacking are usually implicit, even where quantification is explicit [5].

We use existential quantification of contexts to implement variant ownership. This solution is uniform and clearly related to its theoretical underpinnings; typing and the underlying mechanisms are refelcted in the syntax. Furthermore, in combination with type parameterisation, it is extremely expressive.

Outline. In Sect. 2 we give an example explaining and motivating Jo $\exists$. We present $\mathrm{Jo}_{\mathrm{O}} \exists$ in Sect. 3 and $\mathrm{J}_{\mathrm{o}} \exists_{\text {deep }}$ in Sect. 4. We discuss these languages in Sect. 5 and their relation to related work in Sect. 6. We conclude in Sect. 7 


\section{Example}

In this example we use a sugared syntax 1 , rather than the verbose Jo $\exists$ syntax, with implicit packing and unpacking of existential types. Such implicit packing and unpacking appears, for example, in Java wildcards; mapping from the sugared version to $\mathrm{Jo} \exists$ is simple [4. We use $\circ \rightarrow[\mathrm{a}$ b] to denote that the formal context parameter o has the lower bound a and upper bound $\mathrm{b}$, that is, any instantiation of o must be inside $\mathrm{b}$ and outside $2 \mathrm{a}$ in the ownership hierarchy.

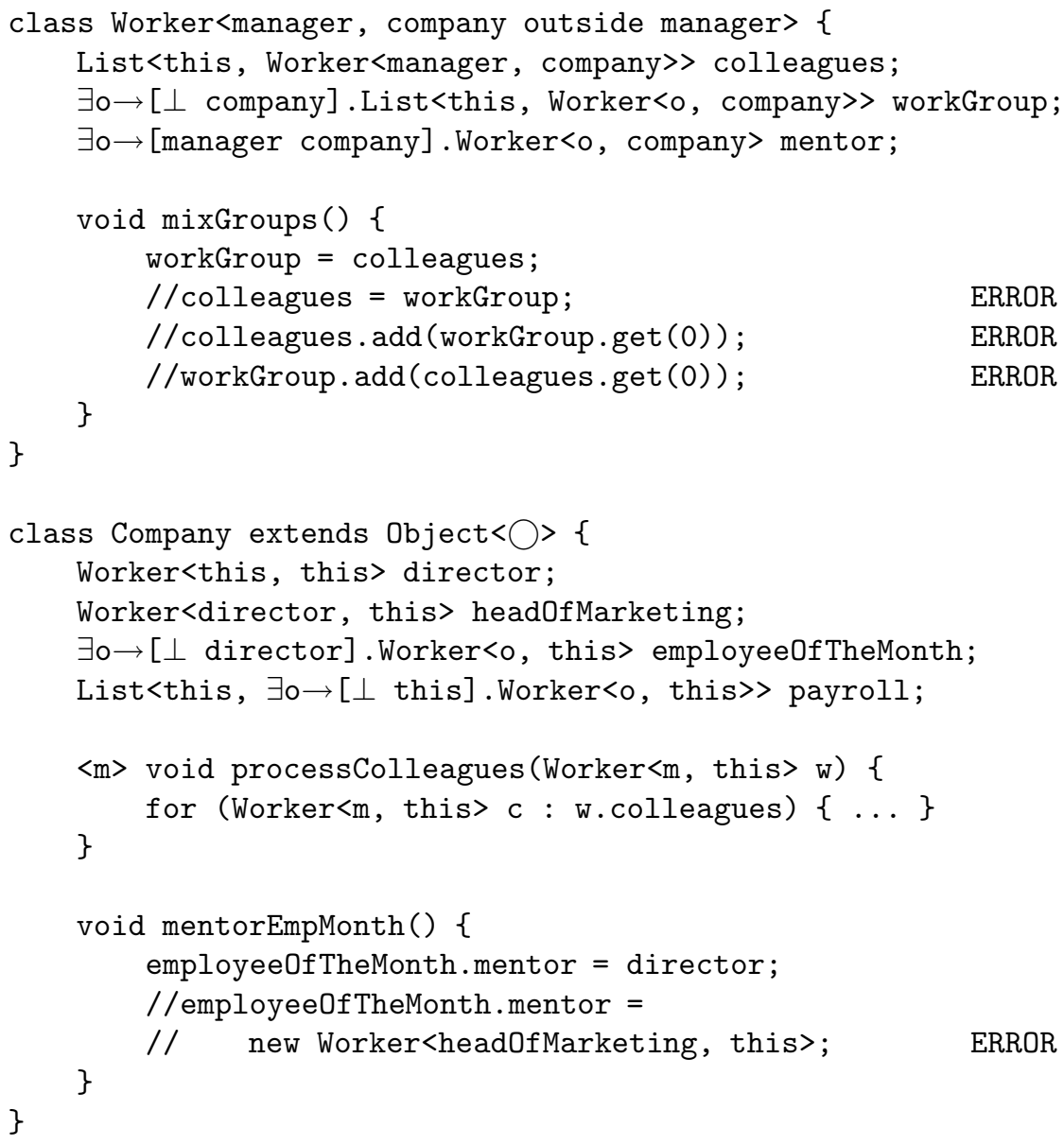

Our example is part of a human resources system for a large company. Each worker in the company is owned by its manager; the employees form a hierarchy with the director at its root. In the Worker class, each worker keeps a list of his

\footnotetext{
${ }^{1}$ We also use fields as context parameters. This is not implemented in Jo $\exists$, but is a relatively easy extension. It is present in, for example, MOJO 6].

${ }^{2}$ We say o outside $o^{\prime}$ to mean $o^{\prime}$ inside $o$.
} 
colleagues. Each colleague is a Worker with the same manager as this. In the Company class, we store references to the director and the head of marketing, whose immediate manager is the director.

So far, we have only used features present in classical ownership types systems. We use existential types where the precise owner of objects is unknown and highlight the features listed in the previous section, e.g., 1. In the Worker class, mentor is some worker who either works with or indirectly manages that worker, but whose exact position in the management hierarchy is not specified (3). A worker may work with some other team of workers in the company (a team is assumed to have a single manager). For example, an engineer may have contact with the management team. This group (workGroup) may have any manager in the company, and this is represented by the existential type. Since we assume all members of the group have the same owner, the existential quantification is outside the List (4).

In the Company class, the employeeOfTheMonth may be any Worker in the company, her manager is not important. The payroll keeps track of every worker in the company. Each worker on the payroll may have a different manager.

The method processColleagues takes a worker (w) as a parameter and performs some action on each of his colleagues. Since the method is polymorphic in the manager (m) of w, we can name $\mathrm{m}$ as the owner of w's colleagues, c (2).

In mixGroups we can set workGroup to colleagues because manager (the manager of colleagues) is within the bounds specified in the type of workGroup. We cannot set colleagues to workGroup, nor add an element of colleagues to workGroup, because workGroup may have any manager, not necessarily this. Even though we can set workGroup to colleagues, we cannot add an element of colleagues to workGroup because although the owner of the workGroup may be any owner, it is a specific owner and not necessarily manager 3 .

Owners-as-dominators. Even in a deep ownership system it can be safe and desirable to support subtype variance. A Worker instance and his mentor (though not his workGroup) satisfy owners-as-dominators in $\mathrm{Jo}_{\mathrm{J}} \exists_{\text {deep }}$. mentorEmpMonth sets the mentor of the employeeOfTheMonth to the director. This preserves owners-as-dominators since the director must transitively manage (own) the employee0fTheMonth, no matter who that is. Setting the employee0f TheMonth's mentor to a new worker owned by the headOfMarketing would violate ownersas-dominators and is not allowed. This is because the employee0fTheMonth may not be transitively owned by this new worker.

\section{$3 \quad \mathrm{Jo} \exists$}

In this section we present the most interesting parts of $\mathrm{J}_{\mathrm{o}} \exists$, a minimal objectoriented language in the style of FGJ [15], with parametrisation of methods and classes by context and type parameters, and existential quantification of contexts. In order to demonstrate ownership properties, we include field assignment

${ }^{3}$ Here, the owner is manager due to the earlier assignment, but in general it will be unknown. 
and a mutable heap. $\mathrm{Jo}_{\mathrm{O}} \exists$ is fully described in the first author's $\mathrm{PhD}$ thesis 4 ] along with much extra detail that could not be included here for space reasons.

Subtype variance in $\mathrm{J}_{0} \exists$ is implemented by existential quantification. Existential types are explicit and are introduced and eliminated (packed and unpacked) using close and open expressions. Thus, we follow the more traditional model of existential types [7], rather than the Java 5.0 approach of using implicit packing and unpacking.

Neither the ownership or existential quantification features of Jo $\exists$ interact with subclassing. Furthermore, the benefits of existential quantification in Jo $\exists$ do not depend on subclassing, nor the absence of subclassing. For these reasons, and because the standard solution to subclassing in ownership types systems is long known 10, we elide subclassing and inheritance. This simplifies the presentation of $\mathrm{J}_{\mathrm{O}} \exists$ and its proofs. $\mathrm{Jo}_{\mathrm{O}} \exists$ could be extended to include subclassing by extending the subtyping and method and field lookup rules following FGJ [15. Subclassing must preserve the formal owner of an object [10]. There are no changes to any of the rules involving quantification.

We are primarily interested in type parameterisation to increase expressiveness of ownership types, rather than to investigate features of generic types. We therefore treat type parameterisation simply and do not support bounds on formal type parameters, nor existential quantification of type variables.

\begin{tabular}{|c|c|c|c|c|c|}
\hline e & $::=$ & $\begin{array}{l}\text { null }|\mathrm{x}| \gamma \cdot \mathrm{f}|\gamma \cdot \mathrm{f}=\mathrm{e}| \gamma \cdot\langle\overline{\mathrm{a}}, \overline{\mathrm{T}} \\
\text { new } \mathrm{C}<\overline{\mathrm{a}}, \overline{\mathrm{T}}>\mid \text { open e as } \mathrm{x}, \overline{\mathrm{o}} \text { in } \mathrm{e} \\
\text { close e with } \mathrm{o} \rightarrow[\mathrm{b} \mathrm{b}] \text { hiding } \overline{\mathrm{a}}\end{array}$ & $\begin{array}{l}\mid \\
\mid \\
\mid\end{array} \mid$ & err & expressions \\
\hline Q & $::=$ & $\operatorname{class} \mathrm{C}<\Delta, \overline{\mathrm{X}}>\{\overline{\mathrm{Tf} ;} \overline{\mathrm{W}}\}$ & & & class declarations \\
\hline W & $::=$ & $<\Delta, \overline{\mathrm{X}}>\operatorname{Tm}(\overline{\mathrm{Tx}})$ \{return $\mathrm{e} ;\}$ & & & method declarations \\
\hline $\mathrm{v}$ & $::=$ & close $v$ with $\overline{\mathrm{o} \rightarrow[\mathrm{b} \mathrm{b}]}$ hiding $\overline{\mathrm{r}}$ & $|\iota|$ & null | err & values \\
\hline $\mathrm{N}$ & $::=$ & class types & $\mathrm{a}$ & $::=0 \mid \mathrm{x}$ & contexts \\
\hline $\mathrm{R}$ & $::=$ & runtime types & $r$ & $::=\bigcirc \mid \iota$ & runtime contexts \\
\hline M & $::=$ & $\mathrm{N} \mid \mathrm{X} \quad$ non-existential types & $\mathrm{b}$ & $::=\mathrm{a} \mid \perp$ & bounds \\
\hline $\mathrm{T}$ & $::=$ & types & & & \\
\hline$\Psi$ & $::=$ & $\overline{\mathrm{X} \rightarrow\left[\begin{array}{ll}\mathrm{b}_{l} & \mathrm{~b}_{u}\end{array}\right]} \quad$ type environments & $\mathrm{x}, \mathrm{y}$ & & variables \\
\hline$\Delta$ & $::=$ & $\overline{\mathrm{o} \rightarrow\left[\begin{array}{ll}\mathrm{b}_{l} & \mathrm{~b}_{u}\end{array}\right]}$ context environments & $\mathrm{X}, \mathrm{Y}$ & & type variables \\
\hline$\gamma$ & $::=$ & \begin{tabular}{l|l|l|l}
$\mathbf{x}$ & $\iota$ & $\mathrm{null}$ & vars and addresses
\end{tabular} & o & & formal owners \\
\hline$\Gamma$ & $::=$ & $\overline{\gamma: \mathrm{T}} \quad$ var environments & $\mathrm{C}$ & & classes \\
\hline $\mathcal{H}$ & $::=$ & $\overline{\iota \rightarrow\{R ; \overline{f \rightarrow v}\}}$ & $\iota$ & & addresses \\
\hline
\end{tabular}

Fig. 1. Syntax of Jo $\exists$ 
Syntax. The syntax of $\mathrm{Jo}_{\mathrm{O}} \exists$ is given in Fig. [1. Entities only used at runtime are in grey . Jo $\exists$ includes expressions for accessing variables (x, which includes this) and addresses $(\iota)$, object creation, null (for field initialisation), field access and assignment, method invocation, and packing and unpacking of existential types.

Class and method declarations $(\mathrm{Q}$ and $\mathrm{W})$ are parameterised by context (o) and type (X) parameters. The former have upper and lower bounds (bounds are actual context parameters - not subtype bounds - and limit the bounded formal context to some part of the ownership hierarchy), and so methods and classes are considered to be parameterised by context environments $(\Delta)$. These are mappings from formal context parameters to their bounds $\left(o \rightarrow\left[\begin{array}{ll}b_{l} & b_{u}\end{array}\right]\right)$.

Contexts (a) consist of context variables $(\mathrm{o})$, variables $(\mathrm{x})$ and the world context (the root object), $\bigcirc$. At runtime we may also use addresses. Runtime contexts $(r)$ are restricted to addresses and $\bigcirc$.

Variable environments, $\Gamma$, map variables to their types. Type environments, $\Psi$, map type variables to bounds on a context. Type variables do not have bounds on the types they may take. The bounds contained in $\Psi$ define upper and lower bounds on the owner of actual types. If the lower and upper bounds on the owner of $\mathrm{X}$ are $\mathrm{b}_{l}$ and $\mathrm{b}_{u}$, then for $\mathrm{C}<\mathrm{o}>$ to instantiate $\mathrm{X}$, o must be outside $\mathrm{b}_{l}$ and inside $\mathrm{b}_{u}$. The bounds in $\Psi$ are manufactured by the type system (in $\mathrm{T}$ Class in Fig. 4 and T-Method [4]) and cannot be defined by the programmer. In $\mathrm{Jo} \exists$ and $\mathrm{J}_{\mathrm{o}} \exists_{\text {deep }}$, upper bounds in $\Psi$ are always $\bigcirc$ and, in effect, are never used; however, we keep upper bounds to allow for easy extension. We only use the lower bound to support deep ownership in $\mathrm{J}_{\mathrm{O}} \exists_{\text {deep }}$ (Sect. 4).

To model execution we use a heap, $\mathcal{H}$, which maps addresses $(\iota)$ to records representing objects. Each record contains the type of the object and a mapping from field names to values. Values (v) are addresses or close expressions that pack addresses.

Types in $J_{0} \exists$. The syntax of types in $\mathrm{J}_{\mathrm{O}} \exists$ is given in Fig. 1. Class types (N) are class names parameterised by actual type and context parameters. The first context parameter is the owner of objects with that type. Class types may be existentially quantified by a context environment to give existential types. For example, $\exists$ o. List<o, Animal> denotes a list owned by some owner. For conciseness in examples, we omit bounds and empty parameter lists where convenient.

By combining existential quantification with type parameterisation we can express many interesting and useful types: $\exists$ o. List<o, Animal<this $>>$ denotes a list owned by some unknown owner where each element is an Animal owned by this, while $\exists \circ 1,02$.List<01, Animal<02〉> denotes a list owned by some owner where all elements are owned by the same owner which may be different from the owner of the list, and $\exists \circ 1$.List<૦ $1, \exists \circ 2$. Animal<02〉> denotes a list where each element is owned by some owner and the owner of each element may be different, finally, $\exists$ o.List<o, Animal<o>> denotes a list where each element in the list and the list itself are owned by the same, unknown, owner.

Subtyping and the Inside Relation. The inside relation relates contexts and is defined by the rules given in Fig. 2. We say that $o_{1}$ is inside $o_{2}\left(\Delta ; \Gamma \vdash \circ_{1} \preceq o_{2}\right)$, if $o_{1}$ is transitively owned by $o_{2}$. The inside relation is reflexive, transitive, and 


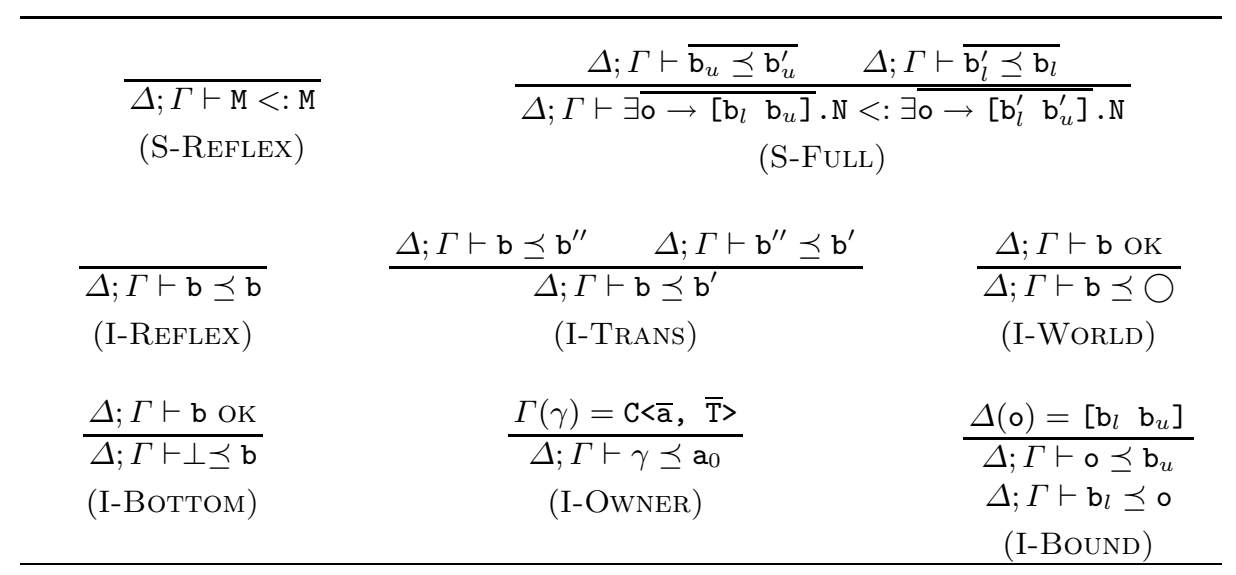

Fig. 2. Jo $\exists$ subtyping, and the inside relation for owners and environments

has top and bottom elements - the world and bottom contexts, respectively. I-OWNER asserts that every variable and address is inside the declared owner of its type (if its type is a class type). For example, if this has type $\mathrm{C}\langle 0\rangle$, then this is inside o. I-Bound gives that a formal context is within its bounds.

Subtyping is also given in Fig. 2. Since there is no subclassing in Jo $\exists$, subtyping of non-existential types is given only by reflexivity. Subtyping between existential types follows the full variant of existential subtyping [147]. Existential types are subtypes where the bounds of quantified contexts in the subtype are more strict than in the supertype.

$$
\begin{aligned}
& \frac{\mathrm{o} \in \operatorname{dom}(\Delta)}{\Delta ; \Gamma \vdash \mathrm{o} \mathrm{OK}} \quad \frac{\Gamma(\gamma)=\mathrm{N}}{\Delta ; \Gamma \vdash \bigcirc \mathrm{OK}} \quad \frac{}{\Delta ; \Gamma \vdash \perp \mathrm{OK}} \quad \frac{}{\Delta ; \Gamma \vdash \gamma \mathrm{OK}} \\
& \text { (F-OWNer) (F-World }) \text { (F-BotTom) (F-VAR) }
\end{aligned}
$$

$$
\begin{aligned}
& \text { class } \mathrm{C}<\overline{\mathrm{O} \rightarrow\left[\mathrm{b}_{l} \mathrm{~b}_{u}\right]}, \overline{\mathrm{X}}>\ldots \quad \Delta ; \Gamma \vdash \overline{\mathrm{a}} \text { OK } \\
& \Delta ; \Gamma \text {, this: } \mathrm{C}<\overline{\mathrm{a}}, \overline{\mathrm{x}}>\vdash \overline{[\overline{\mathrm{a} / \mathrm{o}}] \mathrm{b}_{l} \preceq \mathrm{a}} \quad \Delta ; \Gamma \text {, this: } \mathrm{C}<\overline{\mathrm{a}}, \overline{\mathrm{x}}>\vdash \overline{\mathrm{a} \preceq[\overline{\mathrm{a} / \mathrm{o}}] \mathrm{b}_{u}} \\
& \Psi ; \Delta ; \Gamma \vdash \overline{\mathrm{T}} \text { OK } \quad|\overline{\mathrm{T}}|=|\overline{\mathrm{X}}|
\end{aligned}
$$

(F-Class)

$\frac{\mathrm{X} \in \operatorname{dom}(\Psi)}{\Psi ; \Delta ; \Gamma \vdash \mathrm{X} \mathrm{OK}}$

(F-TYPE-VAR)

$$
\begin{aligned}
& \Delta ; \Gamma \vdash \overline{\mathrm{o} \rightarrow\left[\mathrm{b}_{l} \mathrm{~b}_{u}\right]} \text { OK }
\end{aligned}
$$

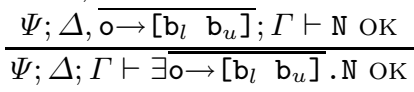

$$
\begin{aligned}
& \text { (F-Exist) }
\end{aligned}
$$

Fig. 3. Jo $\exists$ well-formed contexts and types 
Well-formedness. Well-formed contexts and types are given in Fig. 3. An owner variable is well-formed if it has class type; this guarantees precise information about all unquantified contexts, and that the set of contexts is closed under substitution. This restriction abides by the philosophy of existential types, that abstract packages must be unpacked to be used.

Well-formed class types (F-CLASS) require the class name to have been declared, actual context parameters to be within the bounds of formal context parameters, the number of actual type parameters to match the number of formal type parameters, and actual context and type parameters to be well-formed. Well-formed environments (used in F-ExISTS) are elided, the only interesting aspect is that we require the lower bound of each context variable to be inside its corresponding upper bound.

To check that actual context parameters are within their corresponding bounds, the judging environments are extended with this mapped to $\mathrm{C}<\overline{\mathrm{a}}, \overline{\mathrm{X}}>$, i.e., the class type with actual context parameters and formal type parameters. This is necessary because $\overline{\mathrm{b}_{l}}$ and $\overline{\mathrm{b}_{u}}$ may involve this. We cannot substitute for this, because there may not be a variable or address that contains the object to be substituted. We use a mixture of actual context parameters $(\overline{\mathrm{a}})$ and formal type parameters $(\overline{\mathrm{X}})$ because of the order of application of substitution lemmas in the proofs. Using $\bar{X}$ is safe, even though $\overline{\mathrm{X}}$ are not in scope, because the type parameters of types are never used in the rules defining the inside relation.

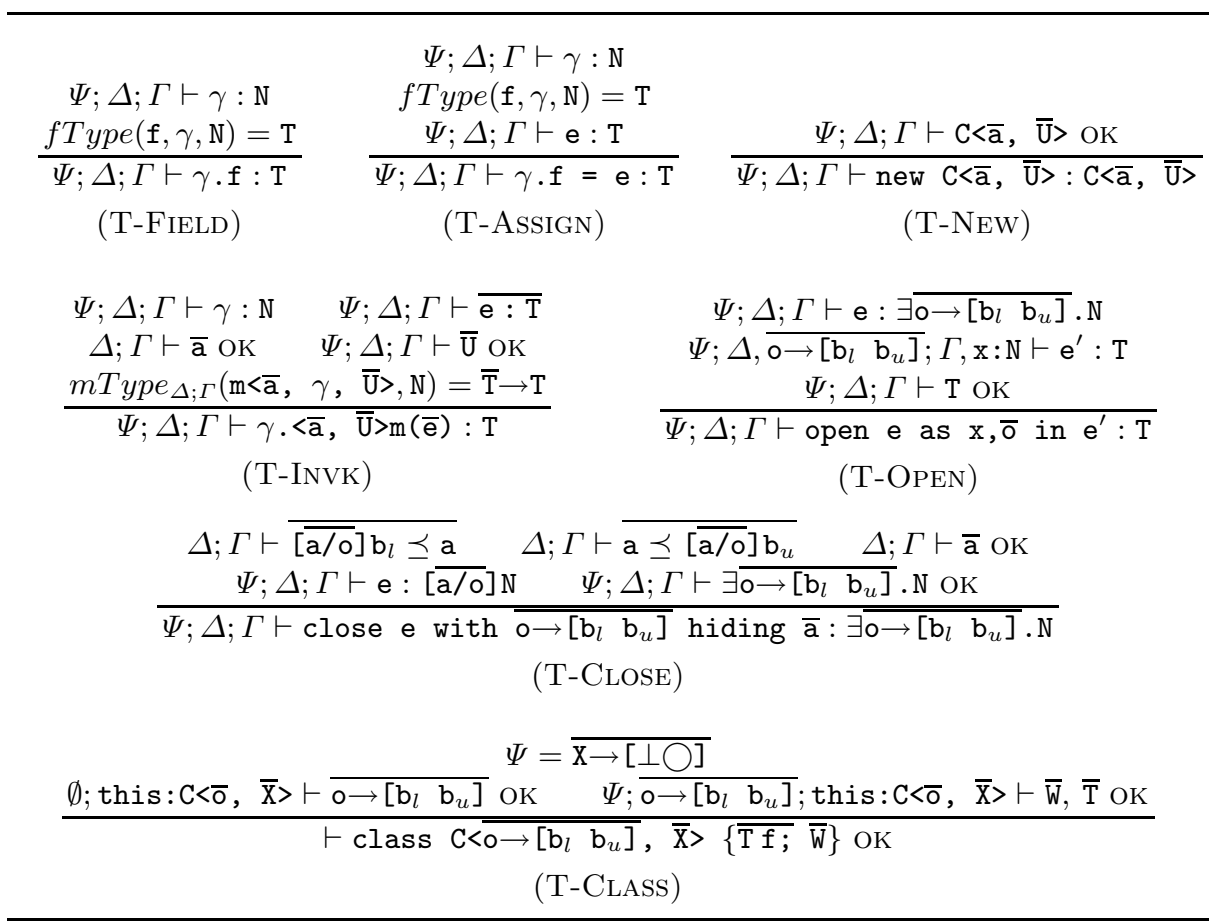

Fig. 4. Jo $\exists$ expression and class typing rules 
Typing. Type rules are given in Fig. 4 Field and variable access (T-FIELD and T-VAR) are close to those of FGJ [15. Field assignment (T-Assign) is a straightforward extension of field access. We adopt the standard subsumption rule (T-SuB). In object creation (T-New), we create uninitialised objects, we do not support constructors. T-NuLL allows null to take any well-formed type. Method invocation is also close to FGJ, with the addition that actual context parameters must be well-formed and within their corresponding formal bounds.

In T-Field, T-Assign, and T-Invk, the receiver is restricted to $\gamma$. This allows us to substitute $\gamma$ for this in field and method types without requiring dependent typing. Expressivity is not lost since the programmer can use an open expression with empty $\bar{o}$ to act as a let expression.

To type check open and close expressions we follow Fun 8 and other classical existential types systems. The type of expression e is unpacked to an owner environment, $\overline{o \rightarrow\left[\begin{array}{ll}b_{l} & b_{u}\end{array}\right]}$, and unquantified type, $N$. We then judge the body of open $\left(\mathrm{e}^{\prime}\right)$ by extending $\Delta$ with $\overline{\mathrm{o} \rightarrow\left[\mathrm{b}_{l} \mathrm{~b}_{u}\right]}$ and adding a fresh variable, $\mathrm{x}$, with type $\mathrm{N}$ to $\Gamma$; $\mathrm{x}$ stands for the unpacked value of e. We ensure no variables escape the scope of the open expression by checking that the result type, $\mathrm{T}$, is well-formed without $\overline{\mathrm{o}}$ or $\mathrm{x}$.

The close expression packs an expression e by hiding some of the context parameters present in e's type. For example, if e has type $C<$ this $>$, then the expression close e with $\circ$ hiding this has the existential type $\exists$ o. C $\langle 0\rangle$.

Example. The assignment employeeOfTheMonth.mentor = director from the example in Sect. 2 is represented with explicit packing and unpacking as:

open employeeOfTheMonth as e,m in

e.mentor $=$ close director with $\circ \rightarrow[\mathrm{m}$ this $]$ hiding this;

Under an environment where e has type Worker $\langle\mathrm{m}$, this $>$, the close and assignment expressions have type $\exists o \rightarrow[\mathrm{m}$ this] . Worker $<0$, this $>$ by T-ClOSE, and by T-Assign and S-Reflex, respectively. By T-Subs, S-Full, and IBтTM, the assignment has the $\mathrm{m}$-free type $\exists \mathrm{o} \rightarrow[\perp$ this $]$.Worker $<0$, this $>$. employeeOfTheMonth (of type $\exists \mathrm{m} \rightarrow[\perp$ director $]$.Worker $<\mathrm{m}$, this $>$ ) can be unpacked as e (of type Worker $<\mathrm{m}$, this $>$ ), used in type checking the assignment, and therefore T-OpEN can be applied, giving the entire expression the type $\exists o \rightarrow[\perp$ this $]$.Worker $<0$, this $>$.

Dynamic Semantics. We elide most of the operational semantics of Jo $\exists$, they are mostly standard 4 . Reduction of open and close expressions is given by the following rule, taken from the classical formulations of existential types [21]:

$\overline{\left.\text { open (close } \mathrm{v} \text { with } \overline{\mathrm{o} \rightarrow\left[\mathrm{b}_{l}\right.} \mathrm{b}_{u}\right]}$ hiding $\overline{\mathrm{r}}$ ) as $\mathrm{x}, \overline{\mathrm{o}}$ in $\mathrm{e} ; \mathcal{H} \leadsto[\overline{\mathrm{r} / \mathrm{o}}, \mathrm{v} / \mathrm{x}] \mathrm{e} ; \mathcal{H}$

The open and close sub-expressions are eliminated, leaving the body of open (e) with formal variables replaced by the packed value and hidden contexts. For example, open (close 3 with o hiding 2 ) as $x, o$ in (this. $\langle 0\rangle m(x)$ ), where 2 and 3 are addresses, reduces to this. $<2>\mathrm{m}$ (3) (we replace $\mathrm{x}$ by 3 and $\circ$ by 2 ).

${ }^{4}$ Object creation, performed in R-NEw, creates a new object with all its fields set to null; i.e., we do not support constructors. 


\begin{tabular}{|c|c|}
\hline $\begin{array}{l}{\operatorname{fType}(\mathrm{f}, \iota, \mathrm{C}<\overline{\mathrm{r}}, \overline{\mathrm{T}}>)=\mathrm{T}^{\prime}}^{\operatorname{lof}} \quad \emptyset ; \Delta ; \mathcal{H} \vdash \overline{\mathrm{v}: \mathrm{T}^{\prime}} \\
\forall \mathrm{v} \in \overline{\mathrm{v}}: \operatorname{add}(\mathrm{v}) \text { defined } \Rightarrow \operatorname{add}(\mathrm{v}) \in \operatorname{dom}(\mathcal{H})\end{array}$ & $\begin{array}{c}\Delta \vdash \mathcal{H} \text { OK } \\
\forall \iota \in f v(\mathrm{e}): \iota \in \operatorname{dom}(\mathcal{H})\end{array}$ \\
\hline $\begin{array}{c}\Delta \vdash \mathcal{H} \text { OK } \\
(\text { F-HEAP })\end{array}$ & $\begin{array}{l}\Delta ; \mathcal{H} \vdash \mathrm{e} \text { OK } \\
(\mathrm{F}-\mathrm{CONFIG})\end{array}$ \\
\hline
\end{tabular}

Fig. 5. Jo $\exists$ well-formed heaps and configurations

In Fig. 5 we give the definitions of well-formed heaps and configurations. Most premises are standard. We insist that the address of all referenced values are in the domain of the heap. The address of a value is given by the partial function add, defined as:

$$
\operatorname{add}(\mathrm{v})= \begin{cases}\iota, & \text { if } \mathrm{v}=\iota \\ \text { add }\left(\mathrm{v}^{\prime}\right), & \text { if } \mathrm{v}=\text { close } \mathrm{v}^{\prime} \ldots \\ \text { undefined, } & \text { otherwise }\end{cases}
$$

which recursively unwraps abstract packages, returning the address within. Thus, $a d d(v)$ is defined if $\mathrm{v}$ is neither null nor null wrapped in a close expression.

Type Soundness. Type soundness in $\mathrm{J}_{\mathrm{O}} \exists$ guarantees that the types of variables accurately reflect their contents, including ownership information. Furthermore, the ownership hierarchy defined statically in a program describes the heap when that program is executed. Although these properties do not constitute an encapsulation property, they are necessary when using ownership information to reason about programs, for example using effects 10. We show type soundness for $\mathrm{Jo}_{\mathrm{O}} \exists$ by proving progress and preservation (subject reduction):

Theorem (progress). For any $\mathcal{H}$, e, T, if $\emptyset ; \emptyset ; \mathcal{H} \vdash$ e : $\mathrm{T}$ and $\emptyset \vdash \mathcal{H}$ oK then either there exists $\mathcal{H}^{\prime}, \mathrm{e}^{\prime}$ such that $\mathrm{e} ; \mathcal{H} \leadsto \mathrm{e}^{\prime} ; \mathcal{H}^{\prime}$ or there exists $\mathrm{v}$ such that $\mathrm{e}=\mathrm{v}$.

Theorem (subject reduction). For any $\Delta, \mathcal{H}, \mathcal{H}^{\prime}, \mathrm{e}, \mathrm{e}^{\prime}, \mathrm{T}$, if $\emptyset ; \Delta ; \mathcal{H} \vdash$ $\mathrm{e}: \mathrm{T}$ and $\mathrm{e} ; \mathcal{H} \leadsto \mathrm{e}^{\prime} ; \mathcal{H}^{\prime}$ and $\Delta ; \mathcal{H} \vdash \mathrm{e}$ OK and $\emptyset ; \mathcal{H} \vdash \Delta$ oK and $\mathrm{e}^{\prime} \neq$ err then $\emptyset ; \Delta ; \mathcal{H}^{\prime} \vdash \mathrm{e}^{\prime}: \mathrm{T}$ and $\Delta ; \mathcal{H}^{\prime} \vdash \mathrm{e}^{\prime}$ OK.

Proofs are given in 4 and can be downloaded from:

http://www.doc.ic.ac.uk/ ncameron/papers/cameron_esop09_proofs.pdf

\section{$4 \mathrm{Jo} \exists_{\text {deep }}$}

$\mathrm{J}_{\mathrm{o}} \exists_{\text {deep }}$ enforces the owners-as-dominators property. It differs from Jo $\exists$ only in its definition of well-formed types, heaps, and classes. We define auxiliary functions to find the owner of an object in the heap $\left(o w n_{\mathcal{H}}(\mathrm{v})\right)$ and the owner of objects with type $\mathrm{T}\left(\mathrm{own}_{\Psi}(\mathrm{T})\right)$ in Fig. 6.

The owner of objects of type $\mathrm{X}$ is the lower bound on the owner of $\mathrm{X}$, recorded in $\Psi$. To find the owner of objects with existential type $(\exists \Delta . \mathrm{C}<\overline{\mathrm{a}}, \overline{\mathrm{T}}>)$, we must 


\begin{tabular}{|c|c|c|}
\hline & $\Psi(\mathrm{X})=\left[\begin{array}{ll}\mathrm{b}_{l} & \mathrm{~b}_{u}\end{array}\right]$ & \\
\hline$\overline{o w n} n_{\Psi}(\mathrm{C}<\overline{\mathrm{a}}, \overline{\mathrm{T}}>)=\mathrm{a}_{0}$ & $\overline{o w n_{\Psi}(\mathrm{x})=\mathrm{b}_{l}}$ & $\overline{o w n}(\exists \Delta . \mathrm{C}<\overline{\mathrm{a}}, \overline{\mathrm{T}}>)=g l b_{\Delta}\left(\mathrm{a}_{0}\right)$ \\
\hline $\mathrm{b} \notin \operatorname{dom}(\Delta)$ & & $\Delta(\mathrm{o})=\left[\begin{array}{ll}\mathrm{b}_{l} & \mathrm{~b}_{u}\end{array}\right]$ \\
\hline$g l b_{\Delta}(\mathrm{b})=\mathrm{b}$ & & $\overline{g l b_{\Delta}(\mathrm{o})=g l b_{\Delta}\left(\mathrm{b}_{l}\right)}$ \\
\hline \multicolumn{3}{|l|}{$\mathcal{H}(\iota)=\{\mathrm{C}\langle\overline{\mathrm{r}}, \overline{\mathrm{T}}>\ldots\}$} \\
\hline$o w n_{\mathcal{H}}(\iota)=r_{0}$ & $n_{\mathcal{H}}$ (close $\mathrm{v}$ with & 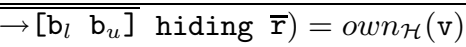 \\
\hline
\end{tabular}

Fig. 6. Owner lookup functions for $\mathrm{Jo}_{\mathrm{o}} \exists_{\text {deep }}$

find a context that is not quantified and that is inside the declared owner of the type $\left(\mathrm{a}_{0}\right)$. This is accomplished by the $g l b$ function; $g l b_{\Delta}(\mathrm{b})$ finds the outermost object that is inside $\mathrm{b}$ and not in the domain of $\Delta$.

The owners-as-dominators property manifests itself as an extra constraint on well-formed heaps; thus, we extend F-HEAP (Fig. 5) as follows:

$$
\frac{\forall \iota \in \mathcal{H} \quad \forall \mathrm{v} \in \mathcal{H}(\iota)^{\cdots} \Delta ; \mathcal{H} \vdash \iota \preceq o w n_{\mathcal{H}}(\mathrm{v})}{\Delta \vdash \mathcal{H} \text { OK }}
$$

(F-HEAP)

Similarly, $\mathrm{Jo}_{\mathrm{o}} \exists_{\text {deep }}$ requires some modifications to the well-formedness rules for class types and classes of $\mathrm{J}_{\circ} \exists$ :

$$
\begin{aligned}
& \forall \mathrm{a}_{i} \in \overline{\mathrm{a}}: \Delta ; \Gamma \vdash \mathrm{a}_{0} \preceq \mathrm{a}_{i} \quad \Psi=\frac{\cdots}{\mathrm{X} \rightarrow\left[\mathrm{o}_{0} \bigcirc\right.} \\
& \frac{\forall \mathrm{T}_{i} \in \overline{\mathrm{T}}: \Delta ; \Gamma \vdash \mathrm{a}_{0} \preceq o w n_{\Psi}\left(\mathrm{T}_{i}\right)}{\Psi ; \Delta ; \Gamma \vdash \mathrm{C}<\overline{\mathrm{a}}, \overline{\mathrm{T}}>\mathrm{OK}} \frac{\perp \notin \overline{\mathrm{T}}, \overline{\mathrm{o} \rightarrow\left[\mathrm{b}_{l} \mathrm{~b}_{u}\right]}}{\vdash \operatorname{class} \mathrm{C}<\overline{\mathrm{o} \rightarrow\left[\mathrm{b}_{l} \mathrm{~b}_{u}\right]}, \overline{\mathrm{X}}>\{\overline{\mathrm{T} f} ; \overline{\mathrm{W}}\} \text { OK }} \\
& \text { (F-ClAss) (T-ClAss) }
\end{aligned}
$$

The extra premises in F-CLASS (together with the well-formedness rules for contexts) ensure that only contexts that are outside an object can be formed by substitution of actual for formal parameters in its class. The owner of an object $\left(a_{0}\right)$ is, by definition, outside that object. The first extra premise ensures that the actual context parameters are outside $a_{0}$. The second premise ensures that the owners of any actual type parameters are outside $a_{0}$. Therefore, all types formed by substitution of contexts or types will have an owner outside this.

In T-CLASS we change the way $\Psi$ is created; the lower bounds in $\Psi$ are the formal owner of the class rather than $\perp$. This is required because of the changes we made to F-CLASS. The class declaration class $\mathrm{C}<0, \mathrm{X}>\{\mathrm{C}<0, \mathrm{X}>\mathrm{f} ;\}$ would not type check without this change: otherwise $\mathrm{C}<0, \mathrm{X}>$ would not be well-formed because $o w n_{\Psi}(\mathrm{X})$ could not be derived to be outside $\mathrm{o}$.

The second extra premise in T-CLASS requires that $\perp$ cannot appear as a bound in the formal context parameters of the class, nor in any existential types given to fields in the class. The intention is to ensure that the owner of all objects 
referenced by objects of the class (including the hidden owner of objects with existential type) is outside the referring object. Therefore, in the example in Sect. 2, the declaration of workGroup would be illegal in $\mathrm{Jo}_{\mathrm{O}} \exists_{\text {deep }}$.

We state the owners-as-dominators property in $\mathrm{J}_{\mathrm{O}} \exists_{\text {deep }}$ as:

\section{Theorem (Owners-as-dominators). For any $\mathcal{H}$, if $\Delta \vdash \mathcal{H}$ oK then $\forall \iota \rightarrow\{\mathrm{R} ;\{\overline{\mathrm{f} \rightarrow \mathrm{v}}\}\} \in \mathcal{H}, \forall \mathrm{v}_{i} \in \overline{\mathrm{v}}: \Delta ; \mathcal{H} \vdash \iota \preceq o w n_{\mathcal{H}}\left(\mathrm{v}_{i}\right)$}

This is given by the added premise to F-HEAP; we prove that this is maintained under execution as part of the proof of subject-reduction [4].

\section{Discussion}

The expressivity of types in $\mathrm{J}_{\mathrm{o}} \exists$ comes from the combination of existential quantification of contexts and type parameterisation. The formalisation of $\mathrm{J}_{\mathrm{o}} \exists$ follows from these starting points and the decision to use explicit packing and unpacking, which simplifies the type rules and proofs for $\mathrm{J}_{\mathrm{O}} \exists$. The natural and uniform emergence of the calculus is reassuring.

Allowing packed values to be be values (and thus stored in the heap) follows earlier work [82114] on existential types and is a natural consequence of explicit packing. However, the owners-as-dominators property is usually phrased assuming that all values are objects (addresses in $\mathrm{J}_{\mathrm{O}} \exists$ ). We must therefore consider how to describe owners-as-dominators in the presence of packed values. We do this by not distinguishing between packed values and the objects that they abstract. This ensures that existential quantification cannot hide violations of owners-as-dominators.

In the type system of $\mathrm{J}_{\mathrm{o}} \exists_{\text {deep }}$, we had to extend the usual restrictions found in ownership systems to enforce owners-as-dominators. Requiring context parameters to be outside an object's owner is standard, we needed to extend this to deal with quantified context variables and type parameters. The crucial observation is that, in enforcing owners-as-dominators, we always wish to show that a value is outside the object that refers to it. It is therefore conservative to use a lower bound on a value's owner rather than the value's owner itself. The additional premises in F-CLASS of $\mathrm{Jo}_{\text {deep }}$ can thus deal with lower bounds on parameters. In the case of quantified context parameters this means that we can use their greatest lower bound. For type parameters we use the lower bound stored in $\Psi$; this motivates using $\Psi$ in $\mathrm{Jo} \exists$ rather than just a set of type variables.

\section{Related Work}

Generics and Ownership Types. Type and ownership information in ownership types systems is usually kept separate 912 25, as in Jo $\exists$. Surprisingly, in OGJ [22], these two kinds of parameters can be expressed using only type parameters. This leads to a small and uniform extension of generic Java that implements deep ownership. The fact that context parameterisation can be encoded using type extension highlights the similarity of the two systems. It will be interesting future work to add Jo $\exists$ 's existential types to OGJ and, it is hoped, reap the benefits of $\mathrm{J}_{\mathrm{o}} \exists$ in a more realistic language. 
Existential Types. Existential quantification of ownership domains in System $F_{\text {own }}$ [17 allows domains to be passed around even if they cannot be named. System $\mathrm{F}_{\text {own }}$ supports existential quantification of types, absent in Jo $\exists$, but does not support subtyping and so existential quantification does not lead to variance.

Infinitary ownership types [9] use existential types to abstract contexts which cannot be named. Because of dynamically created contexts, this is necessary to avoid dependent typing. Existential types in $\mathrm{Jo} \exists$ can be used in the same way. However, since contexts cannot be dynamically created, abstraction is not necessary to avoid dependent typing.

Existential owners can be used in dynamic casts [24]. Casts are not supported in $\mathrm{Jo} \exists$, but they should be straightforward to add. Existential downcasting could then be encoded in $\mathrm{J}_{\mathrm{o}} \exists$ by casting using an existential type.

Variance. Variant ownership types [18] are a programmer friendly way to support use-site subtype variance, and have very similar behaviour to existential types. Jo $\exists$ types are more expressive as they allow lower and upper bounds on contexts (as opposed to upper or lower bounds), type parameters, and explicit quantification (to express types such as $\exists$ o. $\mathrm{C}<\mathrm{o}, \mathrm{o}>$ ).

MOJO [6] uses ? to denote an unknown context parameter. This corresponds to an existentially quantified context bounded by $\perp$ and $\bigcirc$ in Jo $\exists$. In MOJO, ? may be used as an actual context parameter.

In the case of field access, substitution of ? (not found in other systems such as Wild FJ 20] ) produces a similar behaviour to existential types in Jo $\exists$. To prevent field assignment and method call where ? would appear as a type parameter by substitution (but not where ? is written in the type), strict method and field lookup are used. Likewise in $\mathrm{Jo} \exists$, field assignment or method call where the receiver has existential type is type incorrect. Variant types in MOJO are, therefore, treated in the same way as unbounded existential types in $\mathrm{J}_{0} \exists$.

Universes [13] support limited subtype variance through the any notation. Universe types can be given corresponding types in $\mathrm{J}_{\mathrm{O}} \exists$ : any $\mathrm{C}$ corresponds to $\exists \circ \rightarrow[\perp \bigcirc] . C<0\rangle$, peer $C$ corresponds to $\mathrm{C}\langle\mathrm{O}\rangle$ (where $\mathrm{o}$ is the owner of the class declaration in which the type appears), and rep $\mathrm{C}$ corresponds to $\mathrm{C}\langle$ this $>$. The viewpoint adaptation 5 rules of universes correspond to substitution of owners and unpacking and packing in $\mathrm{J}_{\mathrm{O}} \exists$. Generic universes [12] can be described using this correspondence and $\mathrm{J}_{0} \exists$ 's type parameterisation.

An any context is used to facilitate variance in effective ownership [19. During field and method type lookup, all substitutions of any for $\mathrm{x}$ are replaced with substitutions of unknown for $\mathrm{x}$. This mechanism is similar to the abstract contexts of variant ownership types 18 and ? in MOJO. Similarly to these systems, it should be possible to encode the ownership structure of effective ownership in Jo $\exists$. Effective owners (per-method owners) are currently beyond the scope of Jo $\exists$. An effective owner cannot be any, and so there is no variance aspect to these owners.

${ }^{5}$ Viewpoint adaptation is the change in universe annotations when considering a type in a different context from the one in which it was declared. 
In most related work 6 61318, the treatment of unknown contexts is specific to the underlying system; our approach is founded in the theory of existential types and makes clear the relationship between variant types and their behaviour. We discuss in more detail how $\mathrm{J}_{\mathrm{O}} \exists$ can be used to encode and compare the systems described in this section in [4].

\section{Conclusion and Future Work}

$\mathrm{J}_{\mathrm{O}} \exists$ supports context variance in a uniform and transparent fashion using existential types. Expressivity is improved by combining existential quantification of contexts with type parameterisation. We have extended Jo $\exists$ to support ownersas-dominators and proved both versions sound.

Jo $\exists$ can be used to compare and encode ownership systems with different kinds of variance or existential types. Existing mechanisms for supporting context variance have the same behaviour as existential types in $\mathrm{J}_{\mathrm{o}} \exists$ and can be easily encoded (even if other language features cannot). Explicit existential types can give us a clearer picture of the underlying mechanisms used in type checking. Jo $\exists$ can also be used to encode existing kinds of existential types in ownership systems with similar benefits.

We would like to use type parameterisation and context quantification to improve the expressivity of multiple ownership and ownership domains systems, and to investigate how existentially quantified contexts can be used in an effects system. It might be useful to extend $\mathrm{J}_{\mathrm{O}} \exists$ with subclassing, bounds on type variables, and existential quantification of type variables.

Acknowledgement. We would like to thank Werner Dietl and the anonymous reviewers for their detailed and useful feedback, and James Noble for ideas and inspiration from discussions on the MOJO project.

\section{References}

1. Abi-Antoun, M., Aldrich, J.: Ownership Domains in the Real World. In: International Workshop on Aliasing, Confinement and Ownership in object-oriented programming (IWACO) (2008)

2. Armbruster, A., Baker, J., Cunei, A., Flack, C., Holmes, D., Pizlo, F., Pla, E., Prochazka, M., Vitek, J.: A Real-Time Java Virtual Machine with Applications in Avionics. Transactions on Embedded Computing Systems 7(1), 1-49 (2007)

3. Boyapati, C., Rinard, M.: A Parameterized Type System for Race-free Java Programs. In: Object-Oriented Programming, Systems, Languages, and Applications (OOPSLA) (2001)

4. Cameron, N.: Existential Types for Variance - Java Wildcards and Ownership Types. PhD thesis, Imperial College London (2009)

5. Cameron, N., Drossopoulou, S., Ernst, E.: A Model for Java with Wildcards. In: Vitek, J. (ed.) ECOOP 2008. LNCS, vol. 5142, pp. 2-26. Springer, Heidelberg (2008) 
6. Cameron, N., Drossopoulou, S., Noble, J., Smith, M.: Multiple Ownership. In: Object-Oriented Programming, Systems, Languages, and Applications (OOPSLA) (2007)

7. Cameron, N., Ernst, E., Drossopoulou, S.: Towards an Existential Types Model for Java Wildcards. In: Formal Techniques for Java-like Programs (FTfJP) (2007)

8. Cardelli, L., Wegner, P.: On Understanding Types, Data Abstraction, and Polymorphism. ACM Computing Surveys 17(4), 471-522 (1985)

9. Clarke, D.: Object Ownership and Containment. PhD thesis, School of Computer Science and Engineering, The University of New South Wales, Sydney, Australia (2001)

10. Clarke, D.G., Drossopoulou, S.: Ownership, Encapsulation and the Disjointness of Type and Effect. In: Object-Oriented Programming, Systems, Languages, and Applications (OOPSLA) (2002)

11. Clarke, D.G., Potter, J.M., Noble, J.: Ownership Types for Flexible Alias Protection. In: Object-Oriented Programming, Systems, Languages, and Applications (OOPSLA) (1998)

12. Dietl, W., Drossopoulou, S., Müller, P.: Generic Universe Types. In: European Conference on Object Oriented Programming (ECOOP) (2007)

13. Dietl, W., Müller, P.: Universes: Lightweight Ownership for JML. Journal of Object Technology 4(8), 5-32 (2005)

14. Ghelli, G., Pierce, B.: Bounded existentials and minimal typing. Theoretical Computer Science 193(1-2), 75-96 (1998)

15. Igarashi, A., Pierce, B.C., Wadler, P.: Featherweight Java: a Minimal Core Calculus For Java and GJ. ACM Trans. Program. Lang. Syst. 23(3), 396-450 (2001); an earlier version of this work appeared at OOPSLA 1999 (1999)

16. Igarashi, A., Viroli, M.: Variant Parametric Types: A Flexible Subtyping Scheme for Generics. Transactions on Programming Languages and Systems 28(5), 795-847 (2006)

17. Krishnaswami, N., Aldrich, J.: Permission-Based Ownership: Encapsulating State in Higher-Order Typed Languages. In: Programming Language Design and Implementation (PLDI) (2005)

18. Lu, Y., Potter, J.: On Ownership and Accessibility. In: Thomas, D. (ed.) ECOOP 2006. LNCS, vol. 4067, pp. 99-123. Springer, Heidelberg (2006)

19. Lu, Y., Potter, J.: Protecting Representation with Effect Encapsulation. In: Principles of Programming Languages (POPL) (2006)

20. Torgersen, M., Ernst, E., Hansen, C.P.: Wild FJ. In: Foundations of ObjectOriented Languages (FOOL) (2005)

21. Mitchell, J.C., Plotkin, G.D.: Abstract Types have Existential Type. Transactions on Programming Languages and Systems 10(3), 470-502 (1988)

22. Potanin, A., Noble, J., Clarke, D., Biddle, R.: Generic Ownership for Generic Java. In: Object-Oriented Programming, Systems, Languages, and Applications (OOPSLA) (2006)

23. Wrigstad, T.: Ownership-Based Alias Managemant. PhD thesis, KTH, Sweden (2006)

24. Wrigstad, T., Clarke, D.: Existential Owners for Ownership Types. Journal of Object Technology 6(4) (2007)

25. Zhao, T., Palsberg, J., Vitek, J.: Type-based Confinement. J. Funct. Program 16(1), 83-128 (2006) 